\title{
Respuesta a un programa de resiliencia aplicado a padres de niños con Síndrome de Down*
}

\author{
Responding to a program of resilience applied \\ parents of children with Down Syndrome
}

Enviado: noviembre 24 de 2013 | Revisado: octubre 27 de 2014 | Aceptado: noviembre 6 de 2014

\author{
DAVID SÁNCHEZ-TERUEL ** \\ Universidad de Jaén, España \\ María AuXiliadora Robles-Bello *** \\ Asociación Síndrome de Down de Jaén y provincia, España \\ y Universidad de Jaén.
}

\section{doi:10.11144/Javeriana.upsy14-1.rpra}

Para citar este artículo: Sánchez, D., \& Robles, M. A. (2015). Respuesta a un programa de resiliencia aplicado a padres de niños con Síndrome de Down. Universitas Psychologica, 14(2), 645-658. http:// dx.doi.org.10.11144/Javeriana.upsy14-1.rpra

* A la asociación síndrome de Down Jaén y provincia.

** Profesor de Psicología del Departamento de Psicología de la Universidad de Jaén. http://www4. ujaen.es/ dsteruel/Correo electrónico: dsteruel@ ujaen.es

**** Coordinadora Provincial Asociación Síndrome de Down de Jaén y provincia. http://www.downjaen. org Correo electrónico: marobles@ujaen.es

\section{RESUMEN}

El estrés de los padres de niños con Síndrome de Down (SD) sigue siendo un tema de interés para profesionales durante todo el proceso de intervención en atención infantil temprana ( $0-6$ años). El presente estudio pretende comprobar si existen diferencias en 78 padres de niños con Síndrome de Down (entre 3 y 11 meses de edad), con edades comprendidas entre 37 y 56 años $(M=46.5 ; D T=1.94)$, utilizando un entrenamiento basado en el fomento de la resiliencia. Para lograr este objetivo se ha planteado un diseño de evaluación en dos momentos (antes y después de entrenamiento), estableciendo un grupo de padres como experimental y otro como control. Los resultados muestran efectos positivos sobre el estrés que sufren los padres de niños con SD. En particular se reduce el nivel de estrés parental derivado del cuidado del niño (ECN) y el estrés total (ET) tras la aplicación del programa. Se discute la importancia de aplicar tratamientos para el fomento de la resiliencia en padres para amplificar los efectos positivos del proceso de atención infantil temprana en niños con Síndrome de Down y en sus familias.

Palabras clave

atención temprana; Síndrome de Down; padres; resiliencia; estrés

\section{A B S T R A C T}

The stress of parents of children with Down syndrome (DS) remains a handicap of interest to professionals throughout the process of intervention in early child care ( $0-6$ years). This study aims to test whether there are differences in 78 parents of children with Down syndrome (3 to 11 months old), aged between 37 and 56 years ( $M=46.5, S D=1.94)$, using training based on the promotion of resilience. To achieve this goal an evaluation design with two mementos (before and after training) has been done, establishing a group of parents as experimental and another group for control. The results show positive effects on the stress experienced by parents of children with DS. In particular the level of parental stress resulting from reduced childcare derivative (SCC) and total stress (TS) is reduced after the implementation of the program. The importance of applying treatments to promote resilience in parents to amplify the positive effects of early care process in children with Down syndrome and their families is discussed. Keywords:

early intervention; Down syndrome; parents; resilience; stress 
El Síndrome de Down (SD, en adelante) sigue siendo, a nivel mundial, la cromosomopatía de etiología conocida más frecuente en neonatos (Hodapp \& Dykens, 2004; Federación Estatal de Asociaciones de Profesionales de Atención Temprana-FEAPAT, 2008; Organización Mundial de la Salud-O.M.S., 1992, 2013; Robles-Bello, 2007; Robles-Bello \& Sánchez-Teruel, 2011a).

En España los niños y niñas que nacen con alguna discapacidad suelen ser derivados por su pediatra a un Centro de Atención Infantil Temprana (de aquí en adelante CAIT) (Robles-Bello \& SánchezTeruel, 2011b). Son muchos los autores que empiezan a contemplar la necesidad de introducir, en la atención infantil temprana (de aquí en adelante AT), acciones de intervención específicas para los padres y no solo para sus hijos (Cuskelly, Chant, \& Hayes, 2004; Juan, Pérez-López, \& Estévez, 2006; Pérez-López, 2008; 2010). Estos resultados también mostraron el alto nivel de estrés producido por los problemas a los que se enfrentaban los padres de niños con discapacidad, incluso antes de su nacimiento (Gargiulo, 2012; Simmerman, Blacher, \& Baker, 2001). Este aspecto parece influir de manera decisiva sobre un menor índice de desarrollo evolutivo en el proceso temprano de intervención con niños con discapacidad (Flippin \& Crais, 2011; Guralnick, 2005; Hastings \& Taunt, 2002), al igual que en niños y niñas con SD (Hanson, 2004; Skotko $\&$ Canal, 2004). Ejemplos patentes se muestran en trabajos clásicos, donde una intervención temprana prolongada en los niños con SD y en sus familias potencia los beneficios de la intervención (Dmitriev, 1988; Patterson, 1988; 1989; Sharav \& Shlomo, 1986). También en otros estudios más recientes se observaron diferencias en el índice de desarrollo evolutivo, entre el grupo control y experimental de niños con SD en un programa de AT, donde la intervención se centró fundamentalmente en la familia y en la atención a los padres (Guralnick, 2005; Hastings \& Taunt, 2002; Hanson, 2004; VanHoste \& Maes, 2003).

Todo este amplio cuerpo de investigación ha llevado a diversos autores a interesarse por el concepto denominado estrés parental, que se contempla como el grado de malestar consecuencia de los estresores diarios y demandas de cuidado, implícitas en el rol de padre o madre o el grado de ansiedad o malestar que tienen los progenitores a la hora de ejercer su rol como padres o madres (Abidin, 1995; Keller \& Honig, 2004; Pérez-López et al., 2011). Sin embargo, todavía en la práctica terapéutica temprana, el apoyo a la familia en su conjunto y a los padres en concreto, se ha visto descuidado por los profesionales sanitarios y sociales (Córdoba \& Soto, 2007; Dyson, 1986; Quiros, 2009; Patterson, 1988; 1989; Robles-Bello, 2002).

El conocimiento sobre factores de riesgo en la literatura científica sobre discapacidad es más que abundante, pero muy escaso el relacionado con factores que promuevan la protección o resiliencia ante el estrés parental (Poehlmann, Clements, Abbeduto, Farsad, \& Ferguson, 2005). A raíz de investigaciones clásicas sobre resiliencia (Garmezy, 1991; Kobasa, Maddi, \& Kahn, 1982; Kotliarenco, Cáceres, \& Fontecilla, 1997) y otros estudios sobre enriquecimiento personal o resiliencia tras una situación adversa (Martín, Cabrera, León, \& Rodrigo, 2013), se empezó a plantear si podría ser de utilidad el desarrollo de este aspecto en padres y madres de niños con discapacidad (Friborg, Barlaug, Martinussen, Rosenvinge, \& Hjemdal, 2005; Peralta \& Arellano, 2010; Roque, Acle, \& García, 2009). Así, existen pruebas suficientes que indican que el nivel de resiliencia correlaciona negativamente con el nivel de estrés (Masten et al., 1988; Rutter, 1987; Wadsworth et al., 2008). Algunos estudios se han centrado en variables protectoras que promueven un alto nivel de resiliencia en padres de niños con discapacidad, para minimizar el efecto del estrés parental (Grau-Rubio, 2013; Sánchez-Teruel $\&$ Robles-Bello, 2012). De hecho, investigaciones recientes han comprobado que la disminución de los niveles de estrés de los padres, sobre todo de la madre, a través del fomento de su resiliencia contribuye a la buena adaptación del niño, incluso por encima del grado de severidad clínica individual (Farrel \& Krahn, 2014; Gerstein, Crnic, Blacher, \& Baker, 2009; Grant et al., 2013; Fee \& Hinton, 2011; Poehlmann et al., 2005). Sin embargo, tal y como plantean algunos autores, existen escasos estudios donde se apliquen programas de entrenamiento 
para reducir el nivel de estrés en padres con niños con discapacidad y menos aún para incrementar su nivel de resiliencia (Cerqueira da Silva, Dessen, \& Pérez-López, 2012; Roque \& Acle, 2013).

El presente estudio trata de valorar si el nivel de estrés en padres de niños con SD mejora tras la realización de un programa de entrenamiento en resiliencia. Así, se plantea que no existirán diferencias en el nivel de estrés entre ambos grupos (experimental y control) en la situación de preevaluación. En segundo lugar, se espera que existan diferencias en el grupo experimental entre los dos momentos (antes y después) de la aplicación del entrenamiento. Y finalmente, este estudio plantea que no existirán diferencias, tras cuatro meses, en el grupo control entre ambos momentos (antes y después).

\section{Participantes}

La muestra final estuvo formada por 78 padres de niños (entre 3 y 11 meses de edad) con SD, 38 padres y 40 madres con edades comprendidas entre 37 y 56 años $(M=46.5 ; D T=1.94)$. Los criterios de inclusión para participar en este estudio fueron: Tener un hijo o hija con SD (cariotipo Trisomía 21, una de las tres formas de expresión de este síndrome), que además el niño o niña con SD se encuentre en proceso de AT; que los padres cumplimenten el cuestionario de evaluación en los dos momentos (antes y después del entrenamiento); que además asistan a la totalidad de las sesiones de entrenamiento y que firmen el consentimiento informado.

La muestra estuvo inicialmente constituida por 112 padres de niños con SD, de los que 13 personas $(11,61 \%)$ fueron excluidas en la fase pre-valuación porque presentaban errores u omisiones en sus respuestas al cuestionario aplicado y posteriormente 21 personas $(18,75 \%)$ fueron excluidas porque no asistieron a dos o más de dos sesiones en la fase de aplicación del programa de entrenamiento en resiliencia. De ahí, que la muestra de este estudio quedó constituida finalmente por 78 padres de niños con SD. La muestra fue dividida en dos grupos al azar, el primero (experimental) constituido por 40 padres (19 padres y 20 madres) de niños con SD donde se realizó el programa de entrenamiento. El segundo grupo (control o de espera) estuvo constituido por 38 padres (18 padres y 20 madres) de niños con SD, donde no se aplicó dicho programa hasta la finalización de este estudio y solo para aquellos padres que así lo solicitaron. Todos ellos pertenecían a varias asociaciones y grupos de apoyo sobre SD en dos provincias del sur de España. Los padres firmaron un consentimiento informado, en el que quedaban detalladas las bases de este estudio: criterios de inclusión, pruebas aplicadas y objetivos que se perseguían, todo ello en cumplimiento de la Ley de Protección de Datos de Carácter Personal 15/1999 del Gobierno de España. Además, este trabajo fue previamente aprobado por el comité de bioética de la Universidad de Jaén.

\section{Instrumentos}

\section{Hoja de datos sociodemográficos (confeccionada para esta investigación)}

En ella cada participante reflejaba su edad, así como el estado civil (soltero, casado, divorciado/separado, pareja de hecho, viudo), el nivel de estudios (básicos, grado medio/superior, universitarios), estado laboral (desempleado, activo por cuenta ajena, activo por cuenta propia), número de hermanos del niño con SD (hijo único, 1 hermano, 2 o más hermanos).

Índice de estrés parental versión reducida (PSI-SF, Abidin, 1995)

Pretende evaluar el estrés que se experimenta en el ejercicio de la paternidad/maternidad, ya que según Solís y Abidin (1991) esta escala había servido para su propósito, discriminando el estrés entre población con y sin discapacidad. En esta investigación se utilizó el cuestionario de Díaz-Herrero, Brito de la Nuez, López-Pina, Pérez-López \& Martínez-Fuentes (2010), que encontraron tres subdimensiones en la adaptación al español de esta escala (PSI-SF de Abidin, 1995): Estrés derivado del cuidado del niño (ECN), donde se mide el nivel de estrés en los padres debido a las características y el cuidado de los niños. Malestar personal (MP): que mide el estrés 
de los padres debido a características personales que pueden poner en peligro el desempeño adecuado de su rol parental. Y finalmente una escala de estrés total (ET). La consistencia interna (alfa de Cronbach) de dichas subdimensiones fue elevada (Estrés derivado del cuidado del niño: 0.90; Malestar personal: 0.87, y 0.91 para el ET).

Programa de entrenamiento en Resiliencia para cuidadores de personas en situación de dependencia (ERC, Sánchez-

Teruel, \& Robles-Bello, 2012)

Este entrenamiento se desarrolla a lo largo de doce sesiones de 90 minutos una vez por semana. Los aspectos que se trabajan con los padres son: Información básica sobre cuidar en discapacidad, pensamientos disfuncionales, habilidades sociales, autoestima, focalización en actividades positivas, capacidad de delegación, apoyo social, realización de actividades de ocio personales y en familia y redes de apoyo local y comunitario. Cada uno de los componentes se va trabajando a lo largo de las sesiones, de forma secuenciada, siempre utilizando un formato grupal. Se plantean actividades para casa a través de una metodología inductiva, basada en dinámicas de grupo, herramientas audiovisuales y registros de actividad. Se permitía solo la no asistencia a alguna sesión grupal, que sería recuperada a través de una o varias sesiones individuales. Cada sesión fue implementada por dos terapeutas especializados en discapacidad y manejo de grupos, siempre bajo el modelo de trabajo facilitador y participativo, donde los padres se contemplan como los verdaderos expertos; modelo de trabajo aplicado con éxito a ámbitos educativos (Sánchez-Teruel \& Robles-Bello, 2010) o ámbitos laborales (SánchezTeruel, Peña-Herrera \& Cobos, 2010).

\section{Diseño}

El análisis de la eficacia de la intervención se realizó mediante un diseño de grupo control, con medidas antes y después de la aplicación del tratamiento y consistió en la creación de dos grupos (experimental-control) en los cuales se aplicó una medida pre-tratamiento y tras la aplicación del programa de resiliencia al grupo experimental, se tomaron las medidas post-tratamiento. El análisis estadístico consistió en un análisis de muestras relacionadas. Concretamente se realizó una comparación y diferencia de medias $t$ de las puntuaciones, antes y después del entrenamiento en resiliencia. Para estos análisis se utilizó el paquete estadístico SPSS 19.0.

\section{Procedimiento}

En primer lugar se solicitó la participación en el estudio a todos los padres y madres que tenían hijos con SD en 3 Centros de Atención Infantil Temprana (CAIT) de dos provincias del sur de España. En segundo lugar, la intervención fue llevada a cabo por psicólogas de los propios centros, previamente seleccionadas y con experiencia docente en personas con discapacidad. Además, recibieron una formación previa de 20 horas sobre el modelo, metodología y características del programa, por parte de los autores del Programa de entrenamiento en Resiliencia para cuidadores de personas en situación de dependencia (ERC). Posteriormente, se realizó una explicación breve sobre el proceso de entrenamiento a aquellos padres que voluntariamente deseaban participar en la investigación. Tras la firma del consentimiento informado por los padres o madres de cada unidad familiar, se aplicó el PSI-SF (Díaz-Herrero et al., 2010). Solo podía participar en esta investigación un progenitor de cada unidad familiar. Se recogieron los datos de la evaluación, cuyos resultados se corresponderían con la fase de pre-evaluación. Posteriormente se dividió al azar el total de padres en dos grupos: uno experimental y otro control. Se aplicó el programa de entrenamiento (ERC, Sánchez-Teruel, \& Robles-Bello, 2012-ver ejemplo ANEXO 1) solo al grupo experimental. La aplicación del programa se realizó a lo largo de un cuatrimestre en los centros, mientras sus hijos recibían atención infantil temprana (AT). Los padres recibieron una sesión de 90 minutos semanales de entrenamiento. En cada una de las sesiones se trabajaron distintas aspectos, a través de los materiales audiovisuales, gráficos y textos 
que presenta el programa (ERC, Sánchez-Teruel, \& Robles-Bello, 2012). El ERC utiliza una metodología inductiva y centrada en potencialidades y recursos de los propios padres, para manejar diversas situaciones, tanto inducidas como reales (Sánchez-Teruel \& Robles-Bello, 2012). Finalmente, cuatro meses después del entrenamiento, se volvió a evaluar a los padres de ambos grupos a través del PSI-SF (Díaz-Herrero et al., 2010).

Los resultados obtenidos muestran que no existían diferencias entre los dos grupos que componen la muestra, en relación con la edad $\left(F_{(1.77)}=0.245\right.$; $p=0.616)$ y tampoco en relación al género $\left(\mathrm{C}^{2}{ }_{(3)}\right.$ $=3.428 ; p=.133$ ). Respecto al resto de variables sociodemográficas en los grupos (Tabla 1) los resultados muestran que la mayoría de los participantes estaban casados $(82.05 \%)$ y muy pocos vivían como pareja de hecho (15.39\%), sin embargo no existía relación entre los grupos en esta variable $\left(\mathrm{C}^{2}{ }_{(4)}\right.$ $=6.531 ; p=.137)$. Más de la mitad tenían estudios básicos (64.4\%) o de grado medio o superior (24.35\%), no existiendo relación en esta variable ( $\left.\mathrm{C}_{(2)}^{2}=3.784 ; p=0.119\right)$. La mitad estaban desempleados $(50 \%)$ o trabajaban por cuenta propia (34.62\%), tampoco existía relación en esta variable entre el grupo experimental y el grupo control ( $\left.c_{(2)}^{2}=1.784 ; p=0.167\right)$. Más de la mitad de los padres que participaron en este estudio tenían dos o más hijos además del niño con SD (52.56\%) y un $34.62 \%$ tenían solo un hijo más, no existiendo relación entre los grupos en esta variable $\left(\mathrm{C}^{2}{ }_{(2)}=\right.$ $3.784 ; p=0.813$ )

TABLA 1. Resumen de datos sociodemográficos por grupos

\begin{tabular}{llccccc}
\hline Variables socio-demográficas & Total (\%) & Grupo 1 (Experimental) & Grupo 2 (Control) & $\mathrm{c}^{2}$ & $\mathrm{p}$ \\
\hline \multirow{5}{*}{ Estado civil } & Solteros & 0 & 0 & 0 & & \\
& Casados & $64(82.05 \%)$ & $31(39.74 \%)$ & $33(42.31 \%)$ & & \\
& Separados/divorciados & $2(2.56 \%)$ & $1(1.28 \%)$ & $1(1.28 \%)$ & 6.531 & 0.137 \\
& Viudos & 0 & 0 & 0 & & \\
& Pareja de hecho & $12(15.39 \%)$ & $8(10.26 \%)$ & $4(5.13 \%)$ & & \\
& Básicos & $51(64.4 \%)$ & $24(30.77 \%)$ & $27(33.63 \%)$ & & \\
Nivel estudios & Grado medio/superior & $19(24.35 \%)$ & $11(14.10 \%)$ & $8(10.25 \%)$ & 3.784 & 0.119 \\
& Universitarios & $8(10.25 \%)$ & $5(6.41 \%)$ & $3(3.84 \%)$ & & \\
Estado laboral & Desempleados & $39(50 \%)$ & $21(26.92 \%)$ & $18(23.08 \%)$ & & \\
& Activo por cuenta ajena & $12(15.38 \%)$ & $5(6.41 \%)$ & $7(8.97 \%)$ & 1.784 & 0.167 \\
& Activo por cuenta propia & $27(34.62 \%)$ & $14(17.95 \%)$ & $13(16.67 \%)$ & & \\
Hermanos & Hijo único & $10(12.82 \%)$ & $6(7.69 \%)$ & $4(5.13 \%)$ & & \\
& 1 Hermano & $27(34.62 \%)$ & $12(15.38 \%)$ & $15(19.24 \%)$ & 3.784 & 0.813 \\
Total & 2 o más hermanos & $41(52.56 \%)$ & $22(28.21 \%)$ & $19(24.35 \%)$ & & \\
\hline
\end{tabular}

Fuente: elaboración propia

TABLA 2. Resultados evaluación antes y después de intervención en el grupo experimental

\begin{tabular}{lcccc}
\hline & \multicolumn{3}{c}{ Grupo Experimental } \\
\hline Subdimensiones & ANTES M(DT) & DESPUÉS M(DT) & $t$ & $p$ \\
\hline Estrés derivado del cuidado del niño (ECN) & $48.69(3.84)$ & $28.17(1.83)$ & 11.26 & $0.00^{* *}$ \\
Malestar personal (MP) & $21.20(3.02)$ & $19.20(1.02)$ & 7.02 & $0.05^{\text {ns }}$ \\
\hline Estrés yotal (ET) & $67.69(5.97)$ & $25.29(1.97)$ & 38.90 & $0.00^{* *}$ \\
\hline
\end{tabular}

*Diferencias significativas $(\mathrm{p}<0.05) ; * *$ Diferencias muy significativas $(\mathrm{p}<0.01) ; * *$ Diferencias no significativas $(\mathrm{p}=\mathrm{ns})$ Fuente: elaboración propia 
Los resultados obtenidos en este estudio (Tabla 2) parecen mostrar que existen diferencias significativas entre ambos momentos de medida (antes y después del entrenamiento) en el grupo experimental en las subdimensiones de estrés derivado del cuidado del niño (ECN) y estrés total (ET), mientras que en Malestar personal (MP), si bien existen diferencias, estas no llegan a ser significativas.

Respecto al grupo control (Tabla 3) no existen diferencias significativas entre la evaluación previa y la evaluación posterior tras cuatro meses (sin intervención con el ERC).

Respecto a la existencia de diferencias entre los resultados pre y post en ambos grupos (Tabla 4), parece que se detecta un importante descenso en el nivel de estrés en el grupo experimental, respecto al grupo control, fundamentalmente en ECN $(d=$ $1.2, p<0.01)$ y en menor medida en MP $(d=0.43$, $p=0.05)$. Respecto a la puntuación total de estrés (ET) en el grupo experimental respecto al grupo control, debido a la aplicación del tratamiento, existen diferencias significativas en el post $(d=0.68, p$ $<0.05$ ), siendo más evidente en el Estrés derivado del cuidado del niño (ECN).
Según la Organización Mundial de la Salud, O.M.S. (2013) más de mil millones de personas, un $15 \%$ de la población mundial, padece alguna forma de discapacidad. Con este dato se podría deducir que la discapacidad es o será parte de la vida de la mayoría de las familias contemporáneas, tal y como lo han planteado algunos autores (Farrell \& Krahn, 2014). Sin embargo, en línea con investigaciones previas (Grau-Rubio, 2013; Robles-Bello \& Sánchez-Teruel, 2013), parece ser que la discapacidad dentro de un contexto familiar, presenta un alcance limitado, centrándose solo y exclusivamente en déficits y olvidando potencialidades y recursos que les son propios y les hacen más resilientes.

Esta investigación intenta valorar si el nivel de estrés en padres de niños con SD mejora tras la realización de un programa de fomento de resiliencia, planteando, en primer lugar, que no existirán diferencias en el nivel de estrés entre ambos grupos (experimental y control) en la situación antes del entrenamiento. En segundo lugar, se plantea que existirán diferencias en el grupo experimental entre ambas situaciones (antes y después), tras la aplicación del programa entrenamiento en resiliencia. En

TABLA 3. Resultados evaluación momento 1 y 2 en el grupo control

\begin{tabular}{lcccc}
\hline & & Grupo Control & & \\
\hline Subdimensiones & Momento 1 M(DT) & Momento 2 M(DT) & $\mathrm{t}$ & $\mathrm{p}$ \\
\hline Estrés derivado del cuidado del niño (ECN) & $46.97(4.12)$ & $45.12(2.35)$ & 1.67 & $0.34 \mathrm{~ns}$ \\
Malestar personal (MP) & $20.47(1.27)$ & $20.12(1.21)$ & 1.38 & $0.78 \mathrm{~ns}$ \\
\hline Estrés total (ET) & $69.92(5.97)$ & $67.47(1.97)$ & 2.47 & $0.93 \mathrm{~ns}$ \\
\hline
\end{tabular}

*Diferencias significativas $(\mathrm{p}<.005) ; * *$ Diferencias muy significativas $(\mathrm{p}<0.01)$; Diferencias no significativas $(p=\mathrm{ns})$

Fuente: elaboración propia

TABLA 4. Resultados grupo experimental y control y diferencias entre ambos grupos antes y después

\begin{tabular}{|c|c|c|c|c|c|c|c|c|c|c|}
\hline \multirow[b]{2}{*}{ Subdimensiones } & \multicolumn{2}{|c|}{ Grupo Experimental } & \multicolumn{2}{|c|}{ Grupo Control } & \multicolumn{2}{|c|}{$\begin{array}{c}\text { Diferencia } \\
\text { antes-1 } \\
\text { (ambos grupos) }\end{array}$} & \multicolumn{2}{|c|}{$\begin{array}{c}\text { Diferencia } \\
\text { despues-2 } \\
\text { (ambos grupos) }\end{array}$} & \multicolumn{2}{|c|}{$\begin{array}{c}\text { Tamaño del Efecto } \\
\text { despues-2 (ambos } \\
\text { grupos) }\end{array}$} \\
\hline & $\begin{array}{c}\text { PRE } \\
\text { M(DT) }\end{array}$ & $\begin{array}{l}\text { POST } \\
\text { M(DT) }\end{array}$ & $\begin{array}{l}\text { Evaluación } \\
\text { Momentol } \\
\text { M(DT) }\end{array}$ & $\begin{array}{c}\text { Evaluación } \\
\text { momento2 } \\
\text { M(DT) }\end{array}$ & $\mathrm{t}$ & $\mathrm{p}$ & $\mathrm{t}$ & $\mathrm{p}$ & d & p \\
\hline Estrés derivado del cuidado del niño (ECN) & $48.69(3.84)$ & $28.17(1.83)$ & $46.97(4.12)$ & $45.12(2.35)$ & 3.02 & $0.71 \mathrm{~ns}$ & 1.02 & $0.00 * *$ & 1.2 & $0.00 * *$ \\
\hline Malestar personal (MP) & $21.20(3.02)$ & $19.20(1.02)$ & $20.47(1.27)$ & $20.12(3.21)$ & 2.26 & $0.45 \mathrm{~ns}$ & 1.26 & $0.06 \mathrm{~ns}$ & 0.43 & $0.05 \mathrm{~ns}$ \\
\hline Estrés total (ET) & $67.69(5.97)$ & $25.29(1.97)$ & $69.92(5.97)$ & $67.47(1.97)$ & 4.54 & $0.34 \mathrm{~ns}$ & 0.37 & $0.00 * *$ & 0.68 & $0.03^{*}$ \\
\hline
\end{tabular}

*Diferencias significativas $(\mathrm{p}<0.05)$; **Diferencias muy significativas $(\mathrm{p}<0.01)$; Diferencias no significativas $(\mathrm{p}=\mathrm{ns})$ Fuente: elaboración propia 
tercer lugar, se espera que no existan diferencias en el grupo control entre ambos momentos (situación 1 y 2) tras cuatro meses.

En este estudio no existían relaciones entre los dos grupos de padres que componen la muestra, experimental y control, respecto a la edad, el género y otras variables socio-demográficas. En particular, los padres estaban la mayoría casados, tenían estudios básicos, la mitad se encontraba en situación de desempleo y tenían dos o más de dos hijos, además del niño o niña con SD.

Numerosos estudios han demostrado la importancia de evaluar el estrés paterno y materno dentro del sistema familiar, cuando nace un hijo con discapacidad (Dyson, 1993; Díaz-Herrero et al., 2010; Gargiulo, 2012; Oronoz, Alonso-Arbiol, \& Balluerka, 2007), sobre todo, cuando esa discapacidad tiene un origen claramente genético como es el caso del SD (Calero, Robles-Bello, \& García, 2010). Los resultados de este estudio aportan evidencia a favor de la eficacia, como método de reducción del estrés en padres y madres de niños con SD, para potenciar los factores protectores a través de programas específicos de entrenamiento en resiliencia en este colectivo. Estos resultados van en la línea de estudios anteriores, que demuestran que el nivel de resiliencia correlaciona de forma negativa con el nivel de estrés (Masten et al., 1988; Martín et al., 2013; Rutter, 1987; Wadsworth et al., 2008), y que existen escasos programas de entrenamiento para fomentar la resiliencia en padres de niños con SD (Cerqueira da Silva et al., 2012). El efecto positivo de los programas de incremento de la resiliencia en la lucha contra el estrés paterno y materno puede ser explicado porque se trabajan aspectos cognitivos, emocionales y conductuales que mejoran el proceso de afrontamiento más centrado en las peculiaridades de cada niño con SD, en el entrenamiento y gestión de problemas de conducta específicos y en el fomento de pensamientos y habilidades constructivas en los padres, a través de la puesta en marcha de fuertes redes sociales de apoyo, tal y como lo han planteado estudios anteriores (Grant et al., 2013; Roque \& Acle, 2013).

También este estudio evidencia que el grupo experimental reduce su nivel de estrés tras la in- tervención en resiliencia, sobre todo en la subdimensión denominada estrés derivado del cuidado del niño (ECN). Estos resultados están en relación con otros estudios, donde se plantea que fomentar los aspectos personales y contextuales durante el proceso de crianza y educación de un niño con discapacidad, minimiza los efectos negativos de su cuidado, incluso por encima del grado de severidad clínica individual o de la extensión y severidad de los síntomas del comportamiento del niño (Farrel \& Krahn, 2014).

En esta línea, el manual europeo de diagnóstico destinado a profesionales de atención temprana (FEAPAT, 2008) y algunos estudios (Flippin \& Crais, 2011; Roque, Acle, \& García, 2009; Sánchez-Teruel \& Robles-Bello, 2012) muestran la importancia de trabajar de forma precoz, no solo con niños con discapacidad, sino también con sus padres, para potenciar los aspectos positivos y enriquecedores asociados a los cuidados de sus hijos. Esto producirá una reducción en su nivel de estrés parental y modulará una mejor implementación del proceso de intervención temprana llevado a cabo por el profesional con su hijo. Por lo tanto, durante el proceso de atención infantil es fundamental una detección y reducción de los elevados niveles de estrés en padres de niños con discapacidad, en particular con SD, lo que podría ayudar a prevenir las graves consecuencias de un mal funcionamiento familiar en los progenitores, en las relaciones de pareja y sobre todo en la eficacia del proceso de intervención temprana aplicado para la estimulación del niño o la niña con SD.

Finalmente, se plantea la necesidad de intervenir con este grupo de padres, no solo desde el ámbito de la resiliencia, sino también con programas de intervención dirigidos al manejo del estrés y la ansiedad, tal y como lo han planteado investigaciones previas (Díaz-Herrero, López-Pina, PérezLópez, Brito de la Nuez, \& Martínez-Fuentes, 2011; Pérez-López et al., 2011). Los resultados obtenidos de este estudio muestran que en el grupo control no parecen existir diferencias significativas entre la evaluación previa y la evaluación realizada tras cuatro meses sin intervención con ERC, por lo que parece que el programa ha tenido éxito y ha ser- 
vido para su propósito. También estos resultados muestran que en la situación de pre-entrenamiento, ambos grupos se comportan igual, al no existir diferencias significativas entre ellos. Sin embargo, tras la intervención el grupo experimental mejora significativamente, de forma particular en las subdimensiones de estrés derivado del cuidado del niño (ECN) y estrés total (ET). Se deduce por tanto, la idoneidad del programa ERC para mejorar los niveles de estrés que se experimentan en el ejercicio de la paternidad/maternidad con niños con SD u otras discapacidades intelectuales.

Tal y como lo han planteado estudios anteriores (Candel, 2005; De Linares, \& Pérez-López, 2006) es de interés realizar intervenciones directas sobre los padres de niños que se encuentran dentro de un proceso de atención infantil temprana, esto redundará en una mayor efectividad de esta intervención sobre el propio niño o niña.

Con respecto a las limitaciones del estudio, hay que considerar que a los padres de esta muestra se les solicitó responder sobre la mayoría de problemas de comportamiento o sobre mayores necesidades de apoyo con su hijo o hija. Por lo tanto, los datos sobre los niveles de la conducta problemática y habilidades de adaptación, es probable que reflejen los extremos del grupo y puedan no ser representativos. Por lo tanto, se debe tener cuidado al generalizar a otros padres de niños con SD o con otras discapacidades intelectuales.

El carácter voluntario de la muestra también debe ser considerado, al convertirse la motivación inicial en un factor modulador del éxito en el programa. También hay que destacar la necesidad de llevar a cabo estudios longitudinales a largo plazo, que permitan valorar en qué medida las mejoras obtenidas tras la aplicación del ERC se mantienen en el tiempo. Asimismo sería conveniente realizar estudios en entornos naturales, para desvelar hasta qué punto las mejoras obtenidas por el programa se transfieren realmente a entornos naturales. Todas estas limitaciones plantean perspectivas futuras de estudio para otras investigaciones. En particular, sería conveniente conocer si este programa de fomento de la resiliencia también es efectivo en padres de niños con otras discapacidades intelectuales.
Así como implementar estudios longitudinales y en entornos naturales que valoren la efectividad de la intervención a largo plazo y que midan su grado de generalización.

\section{Referencias}

Abidin, R. R. (1995). Parenting Stress Index (PSI) manual (3rd ed.). Charlottesville, VA: Pediatric Psychology Press.

Calero, M. D., Robles-Bello, M. A., \& García, M. B. (2010). Habilidades cognitivas, conducta y potencial de aprendizaje en preescolares con síndrome de Down. Electronic Journal of Research in Educational Psychology, 8, 87-110.

Candel, I. (2005). Elaboración de un programa de atención temprana. Revista Electrónica de Investigación Psicoeducativa, 7(3), 151-192.

Cerqueira da Silva, S., Dessen, M. A., \& Pérez-López, J. (2012). Evaluación de los servicios de atención a familias de niños con deficiencia. Perspectiva de familiares y profesionales brasileños. Anales de Psicología, 28(3), 866-874. http://dx.doi.org/10.6018/ analesps.28.3.130651

Córdoba, L., \& Soto, G. (2007). Familia y discapacidad: Intervención en crisis desde el modelo ecológico. Psicología Conductual, 15(3), 525-541

Cuskelly, M., Chant, D., \& Hayes, A. (2004, June, 14-19,). Parental stress in fathers and mothers of children with Down syndrome: Are the processes the same? Paper presented at the 12 thWorld Congress of the International Association for the Scientific Study of Intellectual Disability (IASSID). Montpellier, France.

De Linares, C., \& Pérez-López, T. (2006). Programa de intervención familiar. En Pérez-López, J. y G. Brito de la Nuez (Eds.). Manual de Atención Temprana (pp. 353-368). Madrid: Pirámide

Díaz-Herrero, A., Brito de la Nuez A. G., López-Pina, J. A., Pérez-López, J., \& Martínez-Fuentes, M. T. (2010). Estructura factorial y consistencia interna de la versión española del Parenting Stress IndexShort Form. Psicothema, 22(4),1033-1038.

Díaz-Herrero, A., López-Pina, J. A., Pérez-López, J., Brito de la Nuez, A. G., \& Martínez-Fuentes, M. T.(2011). Validity of the Parenting Stress Index- 
Short Form in a Sample of Spanish Fathers. The Spanish Journal of Psychology, 2, 990-997. http:// dx.doi.org/10.5209/rev_SJOP.2011.v14.n2.44

Dmitriev, V. (1988). Cognition and the acceleration and maintenance of developmental gains among children with Down syndrome: Longitudinal data. Down's Syndrome, 11(7), 6-11

Dyson, L. (1986). Stress and adaptation in parents of young handicapped and no handicapped children: a comparative study. Journal of Early Intervention, $1,25-35$.

Dyson, L. L. (1993). Response to the presence of a child with disabilities: Parental stress and family functioning over time. American Journal on Mental Retardation, 98, 207-218.

Farrell, A. F., \& Krahn, G. L. (2014). Family life goes on: Disability in contemporary families. Family Relations, 63, 1-6. http://dx.doi.org/10.1111/fare.12053

Federación Estatal de Asociaciones de Profesionales de Atención Temprana (FEAPAT) (2008). Organización Diagnóstica para la Atención Temprana. Manual de Instrucciones. Madrid: Real Patronato sobre Discapacidad.

Fee, R. J., \& Hinton, V. J. (2011). Resilience in Children Diagnosed with a Chronic Neuromuscular Disorder. Journal of Developmental $\mathcal{E}$ Behavioral Pediatrics, 32(9): 644-650. http://dx.doi.org/10.1097/ DBP.0b013e318235d614

Flippin, M., \& Crais, E. (2011). The need for more effective father involvement early autism intervention. Journal of Early Intervention, 33(1), 24-50

Friborg, O., Barlaug, D., Martinussen, M., Rosenvinge, J. H., \& Hjemdal, O. (2005). Resilience in relation to personality and intelligence. International Journal of Methods in Psychiatric Research, 14, 29-42.

Garmezy, N. (1991). Resiliency and vulnerability to adverse developmental outcomes associated with poverty. American Behavioral Scientist, 34, 416-430.

Gargiulo, R. M. (2012). Special education in contemporary society. An introduction to exceptionality. Belmont, CA: Wadsworth/Thomson Learning

Gerstein, E. D., Crnic, K. A., Blacher, J., \& Baker, B. L. (2009). Resilience and the course of daily parenting stress in families of young children with intellectual disabilities. Journal of Intellectual Dis- ability Research, 53(12), 981-997. http://dx.doi. org/10.1111/j.1365-2788.2009.01220.x

Grant, S., Cross, E., Wraith, J. E., Jones, S., Mahon, L., Lomax, M., ... Hare, D. (2013). Parental social support, coping strategies, resilience factors, stress, anxiety and depression levels in parents of children with MPS III (Sanfilippo syndrome) or children with intellectual disabilities (ID). Journal of Inherited Metabolic Disease, 36, 281-291. http:// dx.doi.org/10.1007/s10545-012-9558-y

Grau-Rubio, C. (2013). Fomentar la resiliencia en familias con enfermedades crónicas pediátricas. Revista Española de Discapacidad, 1(1), 195-212 doi: http:// dx.doi.org/10.5569/2340-5104.01.01.10

Guralnick, M. J. (2005). Early intervention for children with intellectual disabilities: current knowledge and future prospects. Journal of Applied Research in Intellectual Disabilities, 18, 313-324. http://dx.doi. org/10.1111/j.1468-3148.2005.00270.x

Hanson, M. J. (2004). Veinticinco años de Intervención Temprana. Un seguimiento de niños con síndrome de Down y sus familias. Revista Síndrome de Down, 21,42-53.

Hastings, R. P., \& Taunt, H. M. (2002). Positive perceptions in families of children with developmental disabilities. The American Journal on Mental Retardation, 107, 116-127.

Hodapp, R. M., \& Dykens, E. M. (2004). Genética y fenotipo conductual en las discapacidad intelectual: su aplicación a la cognición y a la conducta problemática (1ㄹ- parte) Revista Síndrome de Down, 21, 134-149.

Juan, M. J., Pérez-López, J., \& Estévez, C. (2006). La formación de padres como herramienta de prevención primaria en atención temprana. Revista de Atención Temprana, 9, 95-103

Keller, D., \& Honig, A. S. (2004).Maternal and paternal stress in families with school-aged children with disabilities. American Journal of Orthopsychiatry, 74, 337-348.

Kobasa, S. C. Maddi, S. R., \& Kahn, S. (1982). Hardiness and health: A prospective study. Journal of Personality and Social Psychology, 42, 168-177

Kotliarenco, M. A., Cáceres, I., \& Fontecilla, M. (1997). Estado de arte en resiliencia. Washington: Organización Panamericana de la Salud. 
Martín, J. C., Cabrera, E., León, J., \& Rodrigo, M. J. (2013). La Escala de Competencia y Resiliencia Parental para madres y padres en contextos de riesgo psicosocial. Anales de Psicología, 29(3), 886-896. http://dx.doi.org/10.6018/analesps.29.3.150981

Masten, A., Garmezy, N., Tellegen, A., Pellegrini, D. S., Larkin, K., \& Larsen, A. (1988). Competence and stress in school children: the moderating effects of individual and family qualities. Journal of Child Psychology and Psychiatry, 29(6), 779-914.

Organización Mundial de la Salud OMS (1992). Clasificación Estadística Internacional de Enfermedades y Problemas relacionados con la Salud - CIE-10. Madrid: Mediator.

Organización Mundial de la Salud OMS (2013). Discapacidad y salud. Nota descriptiva N³52. Disponible en: http://www.who.int/mediacentre/factsheets/fs352/es/

Oronoz, B., Alonso-Arbiol, I., \& Balluerka, N. (2007). A Spanish adaptation of the Parental Stress Scale. Psicothema, 19(4), 687-692.

Patterson, J. M. (1988). Families experiencing stress. The Family Adjustment and Adaptation Response Model. Family Systems Medicine, 6(2), 202-237.

Patterson, J. M. (1989). A family stress model: The Family Adjustment and adaptation Response. En C. Ramsey (Ed). The science of family medicine (pp. 95-117). New York: Guilford Press.

Peralta, F., \& Arellano, A. (2010). Familia y discapacidad: Una perspectiva teórico-aplicada del Enfoque Centrado en la Familia para promover la autodeterminación. Electronic Journal of Research in Educational Psychology, 8(3), 1339-1362

Pérez-López, J. (2008). Da estimulação precoce à atenção precoce: novas alternativas de atuaçãoprofissional. Anais do I Simpósio Nacional de Atenção e Estimulação Precoce, 9-20. Curitiba, Brasil.

Pérez-López, J. (2010). Cambios conceptuales y marco teórico en atención temprana: nuevas necesidades de formación. Cadernos de Resumos do Simpósio de Cooperação Binacional - Intervenção familiar e desenvolvimento infantil, 17-18. Bauru, Brasil

Pérez-López, J., Rodríguez-Cano, R. A., Montealegre, M. D. P., Pérez-Lag, M., Perea-Velasco, L. P., \& Botella, L. (2011). Estrés adulto y problemas conductuales infantiles percibidos por sus progenitores. XVIII Congreso Internacional Infad (Roma).

Poehlmann, J., Clements, M., Abbeduto, L., Farsad, V., \& Ferguson, D. (2005). Family experiences associated with a child's diagnosis of Fragile X or Down Syndrome: Evidence for disruption and resilience. Mental Retardation, 43(4), 255-267 doi: http://dx.doi.org/10.1352/0047-6765

Quirós, V. (2009). Nuevo modelo para la atención temprana en Andalucía: directrices y desafíos. Revista Iberoamericana de Educación, 48, 1-8

Robles-Bello, M. A. (2002). La Asociación Síndrome de Down de Jaén como nexo de unión entre la escuela y la Familia. En Robles, M. A., Almazán, L. y Torres, J. A. Síndrome de Down y Educación: Una mirada hacia el futuro (pp. 209-225). Jaén: Asociación Síndrome de Down de Jaén y Fundación Santa María.

Robles-Bello, M. A. (2007). Incidencia y prevalencia del Síndrome de Down. Revista Síndrome de Down, 24, $68-70$

Robles-Bello, M. A., \& Sánchez-Teruel, D. (2011a). ¿Qué es el síndrome de Down? Actualización en Síndrome de Down: Creer para ver. Jaén. Fortalezas Editorial.

Robles-Bello, M. A., \& Sánchez-Teruel, D. (Coord.) (2011b). Evaluación e Intervención en Atención Infantil Temprana: Hallazgos recientes y casos prácticos. Jaén: Publicaciones de la Universidad de Jaén, Huarte de San Juan, Serie Psicología.

Robles-Bello, M. A., \& Sánchez-Teruel, D. (2013). Atención Infantil Temprana en España. Papeles del Psicólogo, 34(1), 3-1.

Roque, H. M. P., Acle, T. G., \& García, M. M. (2009). Escala de resiliencia materna: un estudio de validación en una muestra de madres con niños especiales. Revista Iberoamericana de Diagnóstico y Evaluación Psicológica, 1(27), 107-132

Roque, M. del P., \& Acle, G. (2013). Resiliencia materna, funcionamiento familiar y discapacidad intelectual de los hijos en un contexto marginado. Universitas Psychologica, 12 (3) doi: http://dx.doi.org/10.11144/ Javeriana.UPSY12-3.rmff

Rutter, M. (1987). Psychosocial resilience and protective mechanisms. American Journal of Orthopsychiatry, 57, 316-331. 
Sánchez-Teruel, D., \& Robles-Bello, M. A. (2010). La formación y orientación profesional para el empleo en colectivos de riesgo de exclusión: Un modelo de trabajo innovador. I Congreso Internacional virtual de formación del profesorado. Universidad de Murcia

Sánchez-Teruel, D., Peña-Herrera, M., \& Cobos, E. F. (2010). Un modelo de orientación profesional para el empleo de personas en riesgo de exclusión. I Congreso Nacional sobre Orientación y Empleo: nuevos retos ante el mercado laboral. Úbeda (Jaén). O.A.L. de Políticas Activas de Empleo y Consejería de Empleo de la Junta de Andalucía.

Sánchez-Teruel, D., \& Robles-Bello, M. A. (2012). Resiliencia en el cuidado de personas en situación de dependencia. Jaén: Fortalezas Editorial

Sharav, T., \& Shlomo, L. (1986). Stimulation of infants with Down Syndrome: Long-term effects. Mental Retardation, 24(2), 81-86

Simmerman, S., Blacher, J., \& Baker, B. L. (2001). Fathers' and mothers' perceptions of father in- volvement in families with young children with a disability. Journal of Intellectual and Developmental Disability, 26, 325-338.

Solís, M. L., \& Abidin, R. R. (1991).The Spanish version Parenting Stress Index: A psychometric study. Journal of Clinical Child Psychology, 20(4), 372-378. http://dx.doi.org/10.1207/s15374424jccp2004_5

Skotko, B., \& Canal, R. (2004). Apoyo postnatal para madres de niños con síndrome de Down. Revista Sindrome de Down, 21, 54-71.

Van-Hoste, A., \& Maes, B. (2003). Family Factors in the Early Development of Children With Down Syndrome. Journal of Early Intervention, 25(2), 296-309. http://dx.doi.org/10.1177/105381510302500405

Wadsworth, M. E., Raviv, T., Reinhard, C., W., Brian, S., DeCarlo, C., \& Einhorn, L. (2008). An indirect effects model of the association between poverty and child functioning: The role of children's poverty-related stress. Journal of Loss and Trauma, 13(2), 156-185. 


\section{ANEXO 1}

Ejemplo de una sesión para trabajar "Pensamientos disfuncionales" a través del programa de entrenamiento en Resiliencia para cuidadores de personas en situación de dependencia (Sánchez-Teruel y Robles-Bello, 2012):

- Objetivos: formar a padres en el control de pensamientos disfuncionales. La puesta en práctica serán guiadas por la Psicóloga. A continuación se describe una guía que deberá seguir para que los padres pongan en práctica las técnicas vistas durante el desarrollo del contenido teórico.

- Duración: 2 sesiones de 90 minutos (en dos días distintos durante la semana)

\begin{tabular}{cc}
\hline TIEMPO & TAREA \\
\hline $180 \mathrm{~min}$ & Pensamientos disfuncionales \\
\hline
\end{tabular}

\section{REGLAS BÁSICAS (1ª FASE):}

Intentad aprender las siguientes Reglas Básicas para aprovechar al máximo lo que puede ofrecer la técnica:

1. Resista la natural tendencia a aceptar los pensamientos disfuncionales (PD) como ciertos, por el mero hecho de que "parecen correctos" o "razonables". Examínelos y busque a favor o en contra, explicaciones posibles o inferencias más lógicas.

2. Resista la tentación de actuar con reacciones contraproducentes, como la retirada o la huida o la agresividad. Al actuar de esas formas está dando credibilidad a sus PD y los hace más fuertes. Con lo cual la próxima vez estará más propenso a actuar de esas formas, con lo que se incrementa la probabilidad de sentirse triste, ansioso o con miedo.

3. Tendrás que utilizar esta técnica cuando te sientas mal o vayas a afrontar un acontecimiento o situación estresante. Intentando rebobinar (replay) sobre lo que ha pensado con respecto al suceso concreto.

4. iBasta ya de culpabilizar a los demás o lamentarse!. Intente ponerse a trabajar para analizar y racionalizar más su forma de percibir los acontecimientos neutros o negativos que le puedan ocurrir.

\section{¿CÓMO DETECTAR LOS PENSAMIENTOS DISFUNCIONALES (P.D.)?}

1. Son mensajes específicos y cortos: "no sirvo para nada", "todo me va mal"

2. No importa lo irracional que sea un P.D., casi siempre es creído.

3. Entran de golpe en la mente sin dejar a la persona reflexionar sobre su mensaje oculto y dañino

4. Siempre se ponen en lo peor (si me siento angustiado es que voy a tener una crisis)

5. Son difíciles de controlar y de apartar de nuestra mente

\section{SIEMPRE, SIEMPRE QUE NOS SINTAMOS MAL, ¡AHÍ EXISTE UN P.D.!}

\section{EJERCICIOS PRÁCTICOS}

EJERCICIO 1:A las siguientes autofrases negativas especifica qué tipo de P.D. contienen:

1. "Desde lo de Luisa, nunca he vuelto a confiar en una pelirroja"

2. "Pocas personas de aquí parecen más listas que yo"

3. "Si fueras sexualmente más liberal, podríamos ser un matrimonio más feliz"

4. "Me he matado para criar a estos hijos y mira como me lo agradecen"

5. "Si no estás conmigo, estas contra mi"

6. "Pude haber disfrutado de la excursión si no hubiera estado el pollo quemado"

7. "Me siento deprimido, la vida no tiene sentido"

8. "No puedo luchar contra el sistema"

9. "Tu defecto es que todos los meses te encuentras en un aprieto"

10. "Fue un perdedor desde el primer día que se presentó aquî"

11. "Siempre está sonriéndome pero sé que no me quiere" 
12. "No me importa lo que pienses lo volvería a hacer de nuevo"

13. "No nos vemos desde hace dos días, y creo que nuestra relación se está desmoronando"

14. "No deberías preguntar nunca a la gente cuestiones personales"

EJERCICIO 2: Rodear con un círculo que tipo de PD contienen, pueden ser uno o varios:

1. La lavadora se ha estropeado, M. que tiene unos mellizos con SD que todavía usan pañales, se dice a si misma: "Siempre pasa igual, no lo soporto más, la máquina ya me ha jodido el resto del día":

A. Sobregeneralización

B. Razonamiento emocional

C. Culpabilidad

D. Falacia de control

E. Filtraje

2. P. estaba intentando que su novia fuera más afectuosa. Se irritaba cada noche al volver del trabajo si ella no le preguntaba cómo le había ido el día, o no le prestaba atención inmediata. La culpaba de las riñas y le decía que podía mudarse al Polo Norte porque se comportaba como un témpano, y que estaba solo pendiente del niño con SD:
A. Debería
B. Falacia de justicia

C. Sobregeneralización

D. Visión Catastrófica

E. Culpabilidad

\section{EJERCICIO 3:}

Existen una serie de PD en este autodialogo, debe sacar tanto los tipos de PD que hay como las autofrases que los contienen: (Como orientación hay mínimo tres, aunque se pueden especificar más si se justifica correctamente el porqué): "J. se trastorna fácilmente, no puedes decirle nada. Se molesta por todo. Si tuviera un trabajo decente; en vez de ser solo un vigilante jurado. Sus ahorros son casi nulos, asi qué iquién tiene la culpa de que no podamos arreglar la pocilga de casa que tenemos?........ No ves que no es justo hacernos vivir en estas condiciones y más con un niño con SD...."

\begin{tabular}{cc}
\hline Autofrases Negativas & Tipos de P.D. \\
\hline 1. & 1. \\
\hline 2. & 2. \\
\hline 3. & 3. \\
\hline
\end{tabular}

- DETECTAR TIPOS DE PENSAMIENTOS NEGATIVOS AUTOMÁtICOS PROPIOS

- PENSAMIENTOS RACIONALES O AYUDAS

- PRÁCtICA EN hOJA DE TRABAJO

- SESIONES PARA CASA 
